UCRL-JC-121666

PREPRINT

CONF-95 $11143--1$

\title{
Micro-Electro-Mechanical Systems Projects at Lawrence Livermore National Laboratory
}

\author{
PECEMED \\ J. A. Folta \\ FEB 061996 \\ O STI
}

This paper was prepared for submittal to the

Wescon' 95 Conference

San Francisco, CA

November 9, 1995

August 4, 1995

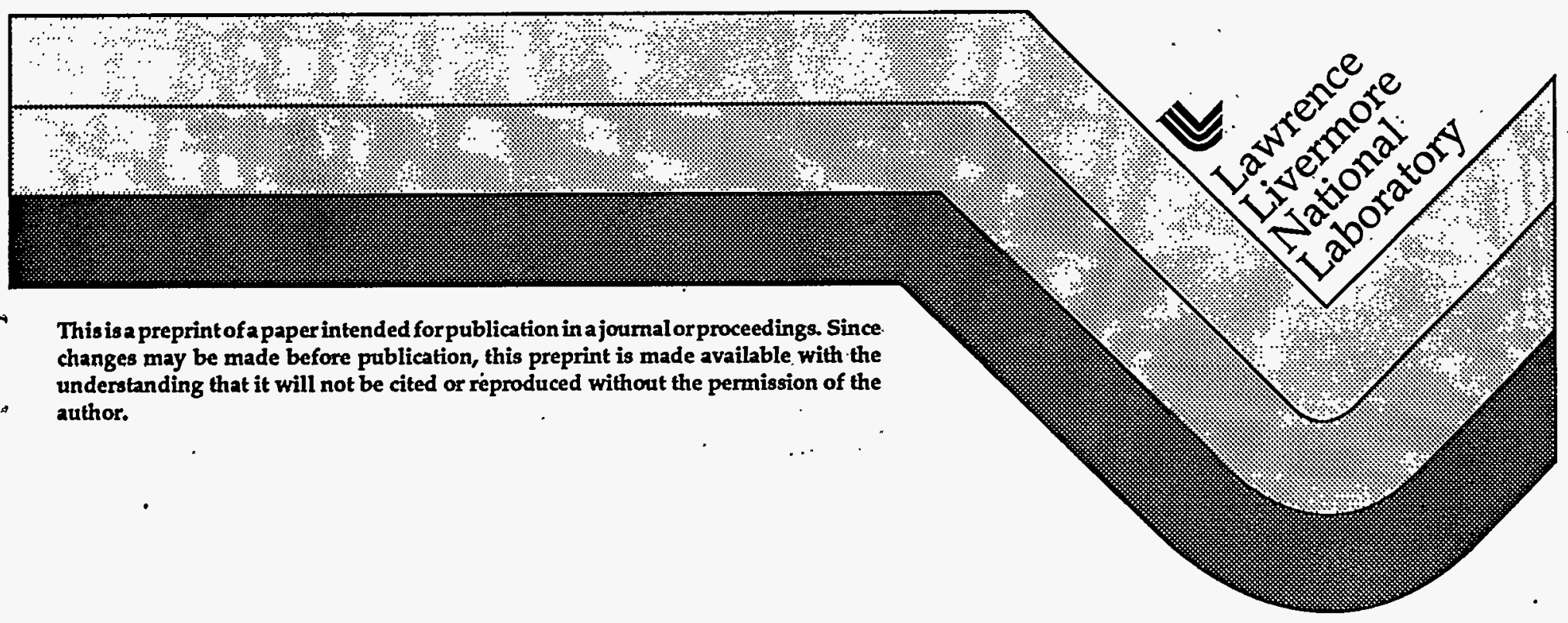




\section{DISCLAIMER}

This document was prepared as an account of work sponsored by an agency of the United States Government. Neither the United States Government nor the University of California nor any of their employees, makes any warranty, express or implied, or assumes any legal liability or responsibility for the accuracy, completeness, or usefulness of any information, apparatus, product, or process disclosed, or represents that its use would not infringe privately owned rights. Reference herein to any specific commercial product, process, or service by trade name, trademark, manufacturer, or otherwise, does not necessarily constitute or imply its endorsement, recommendation, or favoring by the United States Government or the University of California. The views and opinions of authors expressed herein do not necessarily state or reflect those of the United States Government or the University of California, and shall not be used for advertising or product endorsement purposes. 


\section{Micro-Electro-Mechanical Systems Projects at Lawrence Livermore National Laboratory \\ James A. Folta \\ Lawrence Livermore National Laboratory \\ L-222, P.O. Box 808 \\ Livermore, CA 94551}

(510) $422-8354$

The Lawrence Livermore National Laboratory (LLNL) MicroTechnology Center has developed a wide variety of special capabilities used to design, build, and test MEMS (Micro-Electro-Mechanical Systems). Our customers are both the LLNL Programs and a variety of external customers. Typical applications include: custom microstructures for scientific experiments; physical sensors; photonics; miniature tools for catheter-based surgery; and microinstruments for chemical analysis for biomedicine, environment, and treaty verification. The majority of our prototype MEMS devices are fabricated with bulk silicon micromachining, but we also utilize surface micromachining capabilities.

\section{Microvalves}

Lawrence Livermore National Laboratory and Redwood MicroSystems, Inc. (Menlo Park, $\mathrm{CA})$ are working together to expand Redwood's Fluistor ${ }^{T M}$ product line of microfabricated valves. The devices are microfabricated in silicon and are based on Redwood's thermopneumatic actuation principle. The micro-actuator is among the few which provides both high force and displacement needed for valve applications. The actuator motion is precise enough that it can effectively control flows over 6 orders of magnitude. Efforts are currently focused on new generations of valves which are faster, chemically resistant, normally-closed, and compatible with liquids. Future work is planned in integrating arrays of valves with microflow channels, pressure and flow sensors, and chemical detectors to form high performance, microflow systems for pressure regulation, flow control, or chemical analysis. The valves will be optimized for use in analytical chemistry, clinical diagnosis, biotechnology, and environmental monitoring.
Additional applications may include health care, transportation, communications, and chemical processing.

LLNL is providing 3 capabilities to facilitate the microvalve development effort: a fullyequipped microfabrication facility for rapidly building prototype devices; a wide variety of diagnostic instruments for measuring device performance and determining failure mechanisms; and extensive computational facilities for analyzing and simulating device performance. Address inquiries to Jim Folta.

\section{Microflow Systems for Chemical Analysis}

Integration or interconnection of microvalves, capillaries, reactors, and detectors form the basis for portable chemical analysis for environmental, biomedical, and arms control applications. Specific examples include flow injection analysis (FIA) and gas chromatography (GC).

A miniaturized flow injection analysis (FIA) system based upon microvalves can be used for rapid field analysis and in-situ sensing of liquid solutions in the parts per billion range. Small quantities of reagents and sample are intermixed and reacted in a microflow system. Reaction products are detected as they elute from the system. Four primary advantages exist for miniaturized FIA. First, the sensor can be both sensitive and selective. The detector is optimized for sensitivity without regard to selectivity, while the chemical reagents and flow system are optimized to yield high selectivity toward the contaminant of interest. This is in contrast to most chemical sensors in which a tradeoff exists between sensitive and selective detection. Second, analysis times range from 10-100 seconds, allowing up to 300 analyses per hour. Third, the small size of the flow channels minimize the amount of 
reagents required and the volume of waste generated by the analytical procedure. Fourth, FIA is ideally suited for miniaturization because performance improves with reduced size. FIA is robust in the wide range of operating parameters under which one can achieve successful analyses. This allows the microdevice designer wide latitude in selecting among various trade-offs required during system development. Finally, recent advances in microfabricated valves, microreactors, microelectrodes, and optical sources and detectors allow development of such an advanced miniature FIA system. The first microfabricated flow system, including injection valves, reaction chamber, and detection cell, measures $38 \times 25 \times 3 \mathrm{~mm}$. Future generations should be reduced to $20 \times 25 X 3 \mathrm{~mm}$. Address inquiries to Jim Folta.

\section{Gas Chromatography Separation Capillaries}

Microfabricated capillaries have been developed in silicon to allow development of portable GC systems. The capillaries have the circular cross section needed. for high resolution GC separations and are fabricated with isotropic etching followed by aligned wafer bonding. Experimental results show high resolution separation of an injected test sample. Address inquiries to Conrad $\mathrm{Yu}$.

\section{DNA Sequencing by Gel Electrophoresis in Microchannels}

Sequencing of DNA by 'gel electrophoresis is typically performed in slab gel systems. Efforts to increase sequencing rates have generally relied on increasing sample. load capacity (higher lane density or multiple capillary systems) and increasing the electric. field to obtain faster fragment separation (requiring the use of thinner gels or capillaries to reduce heat dissipation). As an alternative to slab and capillary systems, we are investigating a hybrid technique based upon a high density array of electrophoresis channels fabricated using micromachining technologies on a single, large substrate at fixed locations. Furthermore, standard polyacrylamide gel (PAG) compositions can be poured into the channels ....using conventional techniques - without the problem of bubble formation and other defects commonly incurred in PAG filled capillaries.

We have found that electrophoretic resolution is dependent upon the surface finish of the micromachined channels. We have developed and refined fabrication techniques that result in electrophoretic resolution in microchannels comparable to the electrophoresis resolution measured in standard slab gels formed between two flat glass plates. We have obtained resolution that results in accurate base calling to greater than 500 DNA bases per channel $(0.2 \mathrm{~mm}$ deep by $1 \mathrm{~mm}$ wide by $25 \mathrm{~cm}$ long microchannels filled with $6 \%$ PAG). This work was performed under a Cooperative Research and Development Agreement (CRADA) between Perkin-Elmer Corporation, Applied Biosystems Division. Address inquiries to Joe Balch or Courtney Davidson.

\section{Flow Cytometry}

We developed a new physical configuration for the detection of right-angle-scattered (RAS) light using a free aqueous stream in flow cytometry which increases the signal-to-noise ratio, narrows the coefficient of variation for uniformly-sized latex spheres, and greatly eases alignment requirements. The new flowstream waveguide (FSW) technique views the scattered light which is trapped within the optical waveguide of the flow stream in air.

In previous flow cytometers, the RAS light has been viewed perpendicularly to the liquid flow, typically using a high-numerical-aperture (NA) microscope objective lens or fiber optic (1-4). Some of the difficulties associated with this approach are the very limited depth of focus of high-NA optics, and the necessity to align precisely the exact focal point of the lens with the point where the excitation light source intersects the sample flow stream. Our invention is the use of the unconfined aqueous flow stream itself as an optical waveguide. There is no "focal point" for this configuration. Alignment simply requires 
centering the light source on the flow stream; the liquid optical waveguide is then automatically "aligned". - This - approach provides robust, stable light collection. For the collection of elastically-scattered light, another advantage accrues; no light is reflected towards the detector, so no obscuration bar is needed for the collection of right-anglescattered light. Experimental results show the FSW-based scatter detector collects the RAS light from 7 to 10 times more effectively than the conventional high-NA microscope-lens system while providing easier alignment and a smaller coefficient of variation for uniformlysized spheres. Address inquiries to Ray Mariella.

\section{Microreactors for PCR}

We have developed a miniaturized, batteryoperated, bioanalytical instrument for DNA analyses based on the polymerase chain reaction (PCR) with funding by the MEMS program of the Advanced Research Projects Agency (ARPA). PCR is a biochemical reaction used to selectively reproduce DNA to allow detection and identification of targeted DNA molecules. Experimental results show that the thermal cycling unit successfully performs PCR analyses faster than commercially available full-size instruments by taking advantage of the favorable thermal properties of silicon. The application of this micro-reactor technology goes beyond DNA analysis into the applications of high precision, low-power, organic, inorganic, and biochemical micro-chemical reactor development. Advantages are speed, portability and reduced chemical usage. Address inquiries to $M$. Allen Northrup.

\section{MicroTools for Intravascular Surgery}

LLNL is developing microfabricated structures for use in catheter-based surgery. One example is a coil release mechanism needed for repair of brain aneurysms. Utilizing silicon and other materials, we are building submillimeter-sized actuators and actuatorfeedback systems for intravascular therapies. We expect to be able to significantly augment the level of sophistication of intravascular tools such as catheters in order to improve $\Rightarrow$ miaimally invasive medical procedures, and provide increased cost-effective healthcare in the U.S. Address inquiries to M. Allen Northrup.

\section{Conductive Polymer Microactuators}

Thin film conductive polymer/polyimide bimorphic microcantilevers have . been actuated $100 \mu \mathrm{m}$ vertically (out-of-plane) upon the volumetric changes induced by electrochemical doping/undoping of the polymer. The microcantilevers are 200-500 $\mu \mathrm{m}$ in length, 50-100 $\mu \mathrm{m}$ in width, and can be extended from a circularly-curled geometry, and thus generate more than $100 \mu \mathrm{m}$ displacement. The power required to drive these actuators was typically less than $2 \mathrm{~mW}$ with voltages as low as 2-3 volts. Dynamically the microcantilevers have been driven as fast as $1.2 \mathrm{~Hz}$. The polymer was stable for over a week stored in air and light. Residual stress in the polymer film is estimated to be as high as $254 \mathrm{MPa}$, and actuation stresses are as high as $50 \mathrm{Mpa}$. Address inquiries to Abe Lee.

\section{Microgripper}

A silicon-based microgripper with a large gripping force, a relatively rigid structural body, and flexibility in functional design was developed. The normally-closed actuation is generated by $\mathrm{Ni}-\mathrm{Ti}-\mathrm{Cu}$ shape memory alloy (SMA) films. Heating to the relatively low actuation temperature $\left(70^{\circ} \mathrm{C}\right)$ induces stress which deflects each side of the microgripper by up to $55 \mu \mathrm{m}$ for a total gripping motion of $110 \mu \mathrm{m}$. When fully open, the force exerted by the film corresponds to a $13 \mathrm{mN}$ gripping force on the tip of the gripper. Fine alignment, $\mathrm{Au}-\mathrm{Si}$ eutectic bonding, precision sawing, Ni-Ti-Cu SMA deposition, as well as bulk silicon micromachining are the core of the process. The processes, although not standard, do not involve exotic fabrication equipment. All the individual processes could be stream-lined for batch processing and therefore reduce fabrication costs.

For future developments, the outer surfaces of the gripper are being integrated with heaters 
and strain sensors for remote active heating and feedback control Another advantage is the possibility to apply alternative actuation mechanisms on the microgripper structure, either hydraulic or thermal bimorphic. Many creative designs of practical microgrippers can be conceived using this basic process. Address inquiries to Abe Lee.

\section{Ni-Ti-Cu Shape Memory Films}

Mixed-sputter deposition was proven to be a viable method for producing shape memory $\mathrm{Ni}-\mathrm{Ti}-\mathrm{Cu}$ films, providing the compositional control necessary for optimization of SMA properties, and substrate curvature, was shown to be an effective technique for characterizing SMA films. The Ni-Ti-Cu film properties were found to be comparable to the properties of bulk alloys, with transformation temperatures from $20-620^{\circ} \mathrm{C}, \quad 10-130^{\circ} \mathrm{C}$ hysteresis, and up to $330 \mathrm{MPa}$ recoverable stress. The addition of 7 at. \% Cu was shown to make the transformation temperatures less sensitive to titanium content. Films with 51 at.\% Ti have transform near body temperature, making them ideally suited for medical applications. Address inquiries to Peter Krulevitch.

\section{Microfabricated Accelerometers}

We have developed 2 prototype accelerometer components for two extreme ranges of acceleration which have potential applications in smart munitions.

\section{(1) High Sensitivity Microaccelerometer}

We have developed ' a high sensitivity silicon microaccelerometer based on a proof mass and microcoil for use with a fixed magnet. The accelerometer consists of a silicon proof mass supported by four silicon cantilever beams. A gold sensing coil is microfabricated on the proof mass. The silicon structure is placed inside a magnetic field provided by a pair of permanent " $U$ " magnets, and the motion of the proof mass is detected by measuring the voltage generated in the wire coil as it is moved back and forth through the magnetic field. A key concept is that the sensing coil can be made with micron-sized IC-type metal traces and remain very sensitive with small external permanent magnets. For a 483-turn coil in a 3260 Gauss magnetic field, the sensitivity was $2.8 \mathrm{~V} / \mathrm{m} / \mathrm{sec}$. with a resonant frequency of $500 \mathrm{~Hz}$.

We have developed complete mathematical models of the deflection of the cantilever beams for both accelerometers which include the steady state and transient responses to step accelerations and square impulse acceleration driving forces. Address inquiries to Steve Swierkowski.

(2) High g Threshold Accelerometer

We have designed and fabricated a latching accelerometer capable of detecting a threshold of acceleration up to tens of kilo-g's. The device uses two silicon cantilever beams which deflect and latch when subjected to a predesigned threshold acceleration level. It is intended for applications requiring a șingleuse device such as in arming and firing systems and crash sensors. Design requirements included: operation at high-g levels (tens of thousands of g's ); no power required; capable of being read long after the accelerating event; immunity to shocks of short duration; and low cost. These criteria are somewhat different from the specifications for accelerometers used in commercial applications. For instance, most commercial accelerometers, especially those for air bag deployment, operate in the 1-100 $\mathrm{g}$ range.

The accelerometer fabrication relied heavily on the anisotropic etching of (110)-oriented silicon wafers. With the vertical, high-aspectratio features formed with this technique, one can conveniently fabricate cantilever beams which interlock, or latch, when accelerated. The level of acceleration which causes the latching is designed by adjusting the shape of the cantilevers (length, width and thickness) and the size of the proof mass at the end of the cantilevers. Work is currently under way to incorporate a series of these cantilevers to detect a sequence of acceleration pulses. Address inquiries to Dino Ciarlo. 


\section{Microchannel Heatsinks}

Microchannel heatsinks have been built for cooling arrays of laser diodes. The heatsinks can dissipate over $1000 \mathrm{~W} / \mathrm{cm}^{2}$. By comparison, a water cooled copper block typically will dissipate 50 watts $/ \mathrm{cm}^{2}$. The heat sinks are fabricated by etching parallel microchannels into the surface of (110)oriented silicon wafers. The microchannels are $20 \mu \mathrm{m}$ wide, $200 \mu \mathrm{m}$ deep, and $1.5 \mathrm{~mm}$ long with a period of $40 \mu \mathrm{m}$. When water flows through the narrow channels, the very high surface area and thin hydrodynamic boundary layer provide the enhanced heat dissipation. A system packaging method was developed to provide reliable fluidic and electrical interconnects to the heatsinks and lasers. The individual package consists of a thin Pyrex glass sandwiched between two silicon wafers. These packages can then be stacked to increase the area of laser emission. The package allows up to 125 heatsinks to be stacked into an array which emits a $2 \mathrm{~cm} \times 10$ $\mathrm{cm}$ laser beam of $13 \mathrm{~kW}$ peak power at $20 \%$ duty factor. Address inquiries to Dino Ciarlo.

\section{Silicon Optical Microbench Technology}

We have developed silicon optical microbenches with integrated heaters for reflowing solder for easier fiber optic pigtailing of optoelectronic devices. The technology is part of our effort to develop automated techniques to reduce the cost of packaged optoelectronic (OE) devices. In particular, we are building a fiber pigtailing machine which will automatically align a fiber to a laser diode or to a lithium niobate modulator with submicron accuracy in less than five minutes. Silicon microbenches provide the stability required to maintain the sub-micron alignment tolerances necessary for single-mode fiber operation.

A typical microbench is $13 \mathrm{~mm} \times 6 \mathrm{~mm} \times 0.5$ $\mathrm{mm}$ and uses polysilicon heaters with gold contacts directly under the fiber to reflow the solder which holds the fiber in a fixed position. Active feedback can be used to couple laser diodes to fiber with sub-micron accuracies. While the fiber is held in the position that maximizes the optical coupling, current is passed through the heater to reflow the solder; the solder then wicks around the metalized fiber. We typically apply one amp of current for approximately $0.5 \mathrm{sec}$ to reflow the solder. We observe no decrease in the light coupled from the $800 \mathrm{~nm}$ laser diode into the singlemode fiber after the solder has cooled and have achieved up to $65 \%$ optical coupling efficiency with conically tapered fibers.

Our microbench geometries with on-board heaters allow rapid attachment of not only the fiber but also other components to be placed on the microbench. Applying larger and longer duration currents allow solder reflow at locations away from the heaters. Using solders with different melting temperatures and judiciously choosing the order of attachment allows a variety of components to be soldered to the microbench without movement of previously attached components. Generally, components furthest from a heater are attached first using a high current through the heater. Address inquiries to Mike Pocha

\section{Microstructures for Science Experiments}

We provide a wide variety of microfabricated structures for LLNL Physics Programs. Examples include: thin silicon nitride and silicon membranes for use as $\mathrm{x}$-ray windows; Fresnel zone plates; V-grooves for fiber optic alignment; and microflow cells with silicon nitride windows for $x$-ray imaging experiments.

We have etched a variety of blazed diffraction gratings and echelon gratings into silicon wafers. Blazed gratings have a saw tooth profile and can be designed to concentrate a high percentage of the diffracted energy into a certain order. The echelon gratings have a staircase profile and are used in interferometers with no moving parts. Both of these structures are important components of spectrometers and interferometers used in chemical detection systems and $x$-ray diagnostics. The gratings are anisotropically etched in silicon with caustic-based etchants. Control of etchant composition and careful alignment to the silicon crystal provide smooth 
etched surfaces. Etching of surfaces cut slightly off the slow-etching (111) plane provide the blazed gratings. Address inquiries to Dino Ciarlo.

\section{Thin Lens}

In many white-light imaging applications it is desirable to make lenses which are extremely thin. Numerous practical advantages are derived if the lenses are one to two orders of magnitude thinner than conventional refractive lenses. For example, these lens systems provide minimal weight and compactness, and are potentially low cost. Although diffractive lenses built with microfabrication techniques are only a few microns thick, they have severe chromatic aberrations when designed and built with only a single etch depth. We have shown that adding a second etch process to provide 3 etch depths significantly reduces chromatic aberration, which leads to performance suitable for forming images for visual applications (eyepieces, magnifiers, etc.) Address inquiries to Dino Ciarlo.

\section{Acknowledgments}

Many thanks to all in the LLNL MicroTechnology Center whose hard work enabled publication of this paper.

This work was performed by Lawrence Livermore National Laboratory under the auspices of the U.S. Department of Energy Contract No. W-7405-Eng-48. 

Technical Information Department $\cdot$ Lawrence Livermore National Laboratory University of California - Livermore, California 94551

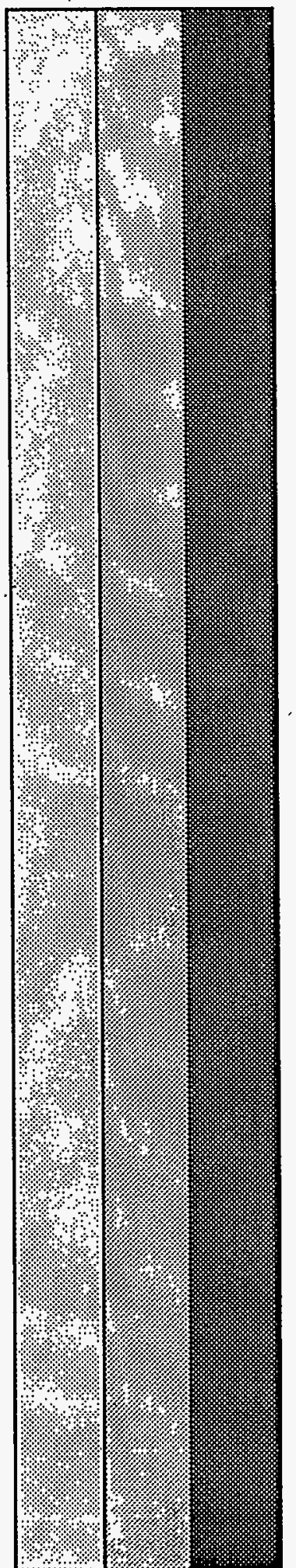

